\title{
Angular compounding by full-channel B-scan modulation encoding for optical coherence tomography speckle reduction
}

Pei Li

Liping Zhou

Yang Ni

Zhihua Ding

Peng Li 


\title{
Angular compounding by full-channel B-scan modulation encoding for optical coherence tomography speckle reduction
}

Pei Li, Liping Zhou, Yang Ni, Zhihua Ding, and Peng $\mathbf{L i}^{*}$

Zhejiang University, College of Optical Science and Engineering, State Key Lab of Modern Optical Instrumentation, Hangzhou, Zhejiang 310027, China

\begin{abstract}
We describe an angular compounding method by full-channel B-scan modulation encoding for speckle reduction in optical coherence tomography. The complex-valued spectral interferogram (SI) is reconstructed by removing one of the conjugate terms in the depth space. Fourier transform of the complex SI along the lateral direction enables a full-channel (with both negative and positive Fourier space) for B-scan modulation in the spatial frequency $(\nu)$ domain. A full-size probe beam, determined by the scanning mirror size, is centered on the mirror pivot, which allows the negative and positive half-channels working in parallel. Compared with the existing method, where only a half-channel (negative or positive) works alone, the proposed full-channel method offers more than twice the performance in speckle reduction. The feasibility of the proposed full-space approach is validated through both the phantom and in vivo human thumbnail experiments. ( 2016 Society of Photo-Optical Instrumentation Engineers (SPIE) [DOI: 10.1117/1.JBO.21.8.086014]
\end{abstract}

Keywords: optical coherence tomography; medical and biological imaging; speckle reduction; angular compounding.

Paper 160269R received Apr. 26, 2016; accepted for publication Aug. 10, 2016; published online Aug. $24,2016$.

\section{Introduction}

Optical coherence tomography (OCT) is a promising imaging modality that provides a noninvasive, high-resolution, and three-dimensional imaging of biological tissues. ${ }^{1}$ It has found a wide range of applications in scientific research and clinics. ${ }^{2,3}$ Like other imaging techniques involving coherence detection such as ultrasound, OCT speckle, caused by coherent addition of many small waves with a random phase and amplitude from randomly placed subresolution scatters, degrades the image quality and limits the identification of detailed structural features that could be crucial for distinguishing normal from diseased tissues. ${ }^{4,5}$ Thus, effective methods of suppressing speckle noise are desired for facilitating the interpretation of OCT images.

A number of methods have been proposed for speckle reduction as discussed in Refs. 4, 6, and 7, which can be generally classified into two categories: software- and hardware-based techniques. The hardware-based approaches are robust in speckle suppression by generating and merging multiple independent subimages. To achieve the subimages with uncorrelated speckle patterns, several diversity mechanisms have been developed by varying the phase of the interference between the subresolution scatters, including frequency, ${ }^{8}$ angular, ${ }^{9,10}$ spatial, ${ }^{11}$ polarization, ${ }^{12}$ and strain. ${ }^{13}$ However, these existing methods are not easily adapted to standard commercial OCT systems.

The angular method illuminates the same collection of subresolution scatters at different angles and the sums of scattering from different incident angles generate uncorrelated speckle patterns. A single-shot angular compounding method has been proposed by encoding different incident/collecting angles into different ranges of path length. Angle-resolved subimages are generated according to the path length and compounded for speckle reduction. ${ }^{9}$ However, to achieve $\sqrt{N}$ times the reduction of speckle noise, the imaging range has to be increased $N$ times, which is unaffordable in most OCT applications. Different incident/collection angles can also be encoded by B-scan modulation frequencies (or Doppler shifts) in the spatial frequency $(\nu)$ domain, and angle-resolved subimages are generated by splitting the modulation spectrum and averaging for speckle reduction. ${ }^{10}$ In the existing modulation encoding method, ${ }^{10}$ to completely separate the complex conjugates in the $\nu$-domain, a small-size probe beam (much less than half of a scanning mirror size) is offset to one side of the mirror pivot, enabling only a half-channel (negative or positive) for modulation. Consequently, there exists a difficult tradeoff between speckle reduction and lateral resolution degradation.

To alleviate the above-mentioned limitations in the existing half-channel approach, we present an angular compounding method by full-channel B-scan modulation encoding for speckle reduction and demonstrate the feasibility through both the phantom and in vivo human thumbnail experiments.

\section{Materials and Methods}

Figure 1 shows the schematic of our spectral domain OCT (SDOCT) system. The light source is a superluminescent diode (SLD) with a central wavelength of $1325 \mathrm{~nm}$ and a full width at half maximum bandwidth of $100 \mathrm{~nm}$, yielding an axial resolution of $\sim 7.7 \mu \mathrm{m}$ in air. In the sample arm, the probe beam was focused on the sample by an objective lens with a focal length of $30 \mathrm{~mm}$. A spectrometer was used to detect the spectral interferogram (SI) of the reference and sample beam, which is equipped with a fast InGaAs linescan camera providing a $147-\mathrm{kHz}$ line-scan rate and 2048 pixels. Only the real part of 


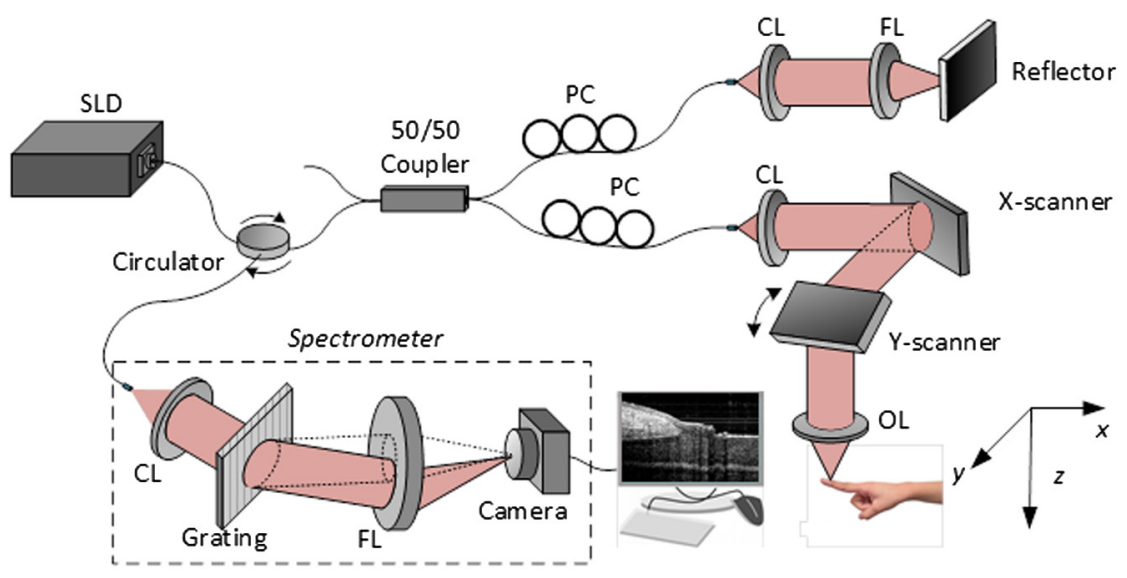

Fig. 1 Schematic of our SDOCT system. SLD, superluminescent diode; PC, polarization controller; FL, focusing lens; $\mathrm{CL}$, collimating lens; $\mathrm{OL}$, objective lens.

OCT complex SI is recorded by the spectrometer. The real-valued raw data of a B-scan is a two-dimensional array $S(k, x)$ with $k$ being the wavenumber of the light source and $x$ being the fast-scan direction. Fourier transform of $S(k, x)$ along the wavenumber $k$-direction generates the reflectivity profile $A(z, x)$ in the depth space (z-space) and the transform along the lateral $x$-direction corresponds to the spatial frequency $(\nu)$ domain. Due to the Fourier transform of real-valued data, both the $z$-space and $\nu$-domain are Hermitian. The spectrometer had a designed spectral resolution of $0.07 \mathrm{~nm}$, offering an imaging range of $\sim 6 \mathrm{~mm}$ on each side of zero delay line in the $z$-space in air.
In the sample arm, the probe beam is incident on a range of positions on the scanning mirror before the objective lens, as depicted in Fig. 2. The light away from the mirror pivot corresponds to an offset $\pm \delta$ and induces a continuous B-scan modulation of the sample arm path length. Thus the phase is induced as the probe beam is scanned in the lateral direction. The modulation frequency $\nu_{m}$ is linearly proportional to the offset $^{14,15}$

$\nu_{m}=\frac{2 k_{0} \delta}{\pi} \omega$
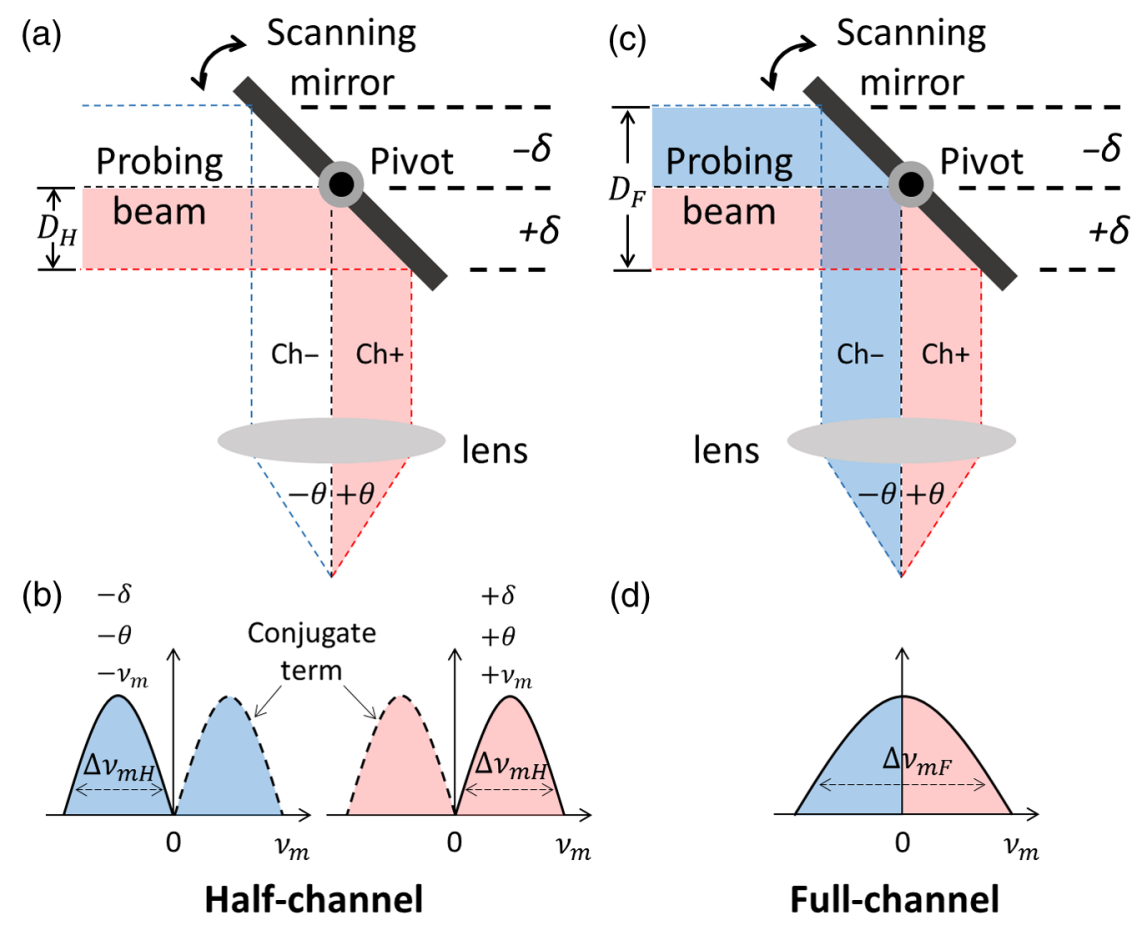

Fig. 2. Schematic of half- (left column) and full-channel (right column) angular compounding. (a), (c) The geometry of sample arms. (b), (d) are the B-scan modulation spectrum $\nu_{m}$ induced by the light offset $\delta$ from the pivot. In the half-channel method, due to the complex-conjugate ambiguity, a half-size probe beam is offset to one side of the pivot, and the total width $\Delta \nu_{m H}$ of its modulation spectrum must not exceed the negative or positive half-space. In the full-channel method, the complex-valued OCT interferogram is reconstructed. A full-size probe beam is centered on the pivot. A wide and conjugate-free modulation spectrum is generated with a width of $\Delta \nu_{m F}$ covering both the negative and positive half-spaces. Theoretically, $\Delta \nu_{m F}=2 \Delta \nu_{m H}$. Ch-, negative half-channel; Ch+, positive half-channel. 
where $k_{0}$ is the central wavenumber of the light source and $\omega$ is the angular velocity of the scanning mirror. The width of the modulation spectrum $\Delta \nu_{m}$ is determined by the diameter of the probe beam $D$

$\Delta \nu_{m}=\frac{2 k_{0} D}{\pi} \omega$

Different incident/collection angles $\pm \theta$ are encoded by different modulation frequencies and angle-resolved subimages are generated by splitting the spectrum and averaging for speckle reduction. The speckle noise is known to reduce in proportion to the reciprocal of the square root of the number of averages or splits $1 / \sqrt{N}$. $^{4}$

As illustrated in Figs. 2(a) and 2(b), the whole channel for B-scan modulation and angular encoding can be roughly divided into two half parts: negative $(\mathrm{Ch}-)$ and positive $(\mathrm{Ch}+)$ half-channels. Ch- corresponds to the negative offset $-\delta$, angle $-\theta$, and modulation $-\nu_{m}$, while $\mathrm{Ch}+$ corresponds to the positive components. In the existing method, ${ }^{10}$ due to the complex-conjugate ambiguity in the $\nu$-domain, one cannot distinguish the positive and negative modulation spectrum and only a half-channel (negative or positive) is enabled with a half-size probe beam offset to one side of the pivot. The half-channel corresponds to a half-space modulation spectrum with a width of $\Delta \nu_{m H}$ in the $\nu$-domain.

Our proposed full-channel method allows the negative and positive half-channels working in parallel, offering more than twice the performance in speckle reduction. As illustrated in Figs. 2(c) and 2(d), a full-size probe beam (both $\pm \delta$ ) is centered on the pivot, inducing both positive and negative half-space modulation spectrums. After background subtraction of the mean spectrum and linearization in $k$-space, the real SI $S(k, x)$ is then processed as presented in Fig. 3. The complex-valued SI $\tilde{S}(k, x)$ is reconstructed by removing one of the conjugate terms in the $z$-space, referring to Figs. 3(a)-3(c) and Ref. 15 for details. A wide and conjugate-free full-space modulation spectrum is generated in the $\nu$-domain through Fourier transform of

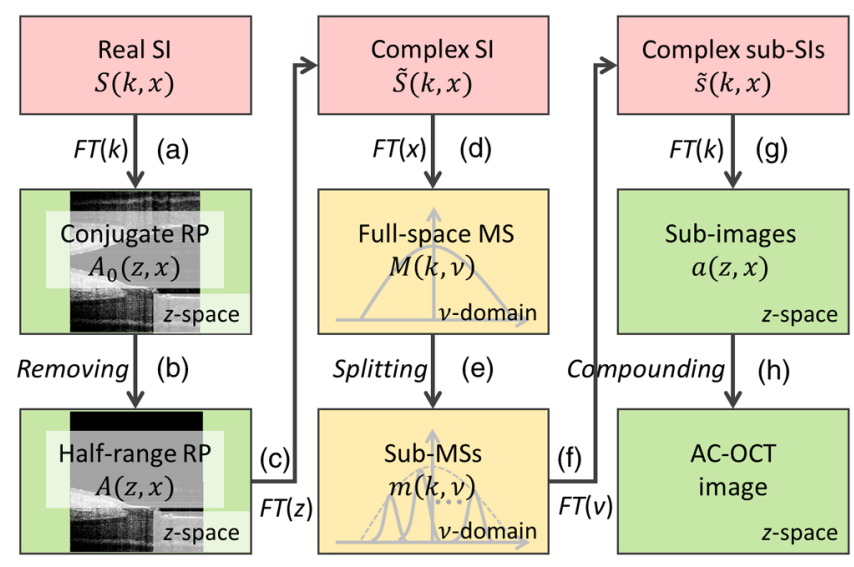

Fig. 3. Flowchart of signal processing in full-channel angular compounding. (a)-(c) Reconstructing complex-valued SI $\tilde{S}(k, x)$ by removing one of the conjugate terms in $z$-space. (d) Generating full-space MS in $\nu$-domain through FT of $\tilde{S}(k, x)$ along the fastscan $x$-direction. (e) Splitting full-space MS for angle-resolved subbands via Gaussian filter banks. (f), (g) Generating and (h) compounding angle-resolved subimages in $z$-space. SI, spectral interferogram; FT, Fourier transform; RP, reflectivity profile, i.e., gray-scale structural image; MS, modulation spectrum.
$\tilde{S}(k, x)$ along the fast-scan $x$-direction, referring to Fig. 3(d). Angle-resolved independent subbands are generated by splitting the full-space modulation spectrum via Gaussian filter banks, ${ }^{15-17}$ referring to Fig. 3(e). The corresponding subimages with different speckle patterns are then generated in the $z$-space by Fourier transform and compounded for speckle reduction, referring to Figs. 3(f)-3(h). The full-channel corresponds to a full-space modulation spectrum with a width of $\Delta \nu_{m F}=2 \Delta \nu_{m H}$ in theory. Assuming a Gaussian shape for the modulation spectrum and a number of $N_{H}$ subbands for each half-channel, the bandwidth of each subband is set as $\Delta \nu_{m H} / N_{H}$, and the filter spacing is the same as the bandwidth of the filter. $1 / e^{2}$ width (the width at $1 / e^{2}$ maximum amplitude of the spectrum) is used here for less overlap between subbands and minimized correlation of speckle pattern. Finally, the full-channel offers twice the number of subbands $\left(2 N_{H}\right)$ over the half-channel.

To demonstrate the feasibility and superiority of our proposed full-channel method, both the half- and full-channel approaches were implemented in the studies of both a homogeneous solid phantom (a mixture of agarose gel and 5\% milk) and in vivo human thumbnail. The use of human subjects was approved by the Institutional Review Board at the Zhejiang University. In the full-channel approach, a full-size collimated probe beam with a diameter of $D_{F}=3.4 \mathrm{~mm}$ was centered on the pivot of the scanning mirror, yielding a lateral resolution $\delta_{x}=14.9 \mu \mathrm{m}$ and a full-space modulation spectrum covering both the negative and positive $\nu$-domain. In the half-channel approach, a small-size beam with a diameter $D_{H}=1 \mathrm{~mm}$ was offset to one side of the pivot, yielding a lateral resolution of $\sim 50.6 \mu \mathrm{m}$ and a half-space modulation spectrum. Such a small beam size is to completely avoid the overlap between the conjugate B-scan modulation spectrums. Each B-frame was composed of 500 A-lines, covering a range of $\sim 3 \mathrm{~mm}$ in the lateral direction.

The parameter of speckle contrast $(C)$ in both the half- and full-channel methods was measured versus the number of splits/averages $N$. The speckle contrast $(C)$ is defined as ${ }^{10}$

$C=\sigma / \mu$,

where $\sigma$ and $\mu$ are the standard deviation and the mean of the OCT amplitudes in the region of interest (ROI), respectively. The measurements of $\sigma$ and $\mu$ are on a linear intensity scale. In this study, the number of splits is equivalent to the number of averages. Assuming the speckle contrast of the original OCT image (without averaging) is $C_{0}$, in theory, $C_{0} \approx 0.52$, and is to reduce in proportion to the reciprocal of the square root of the number of averages ${ }^{4}$

$C=C_{0} / \sqrt{N}$

where $N$ equals to $N_{H}$ in half-channel and $2 N_{H}$ in full-channel, respectively.

Furthermore, the image metrics, such as contrast-to-noise ratio (CNR), average effective number of looks (ENL), were also quantitatively evaluated. The CNR and ENL are defined as $^{18}$

$\mathrm{CNR}=10 \log \left[\left(\mu_{m}-\mu_{b}\right) / \sqrt{\sigma_{m}^{2}+\sigma_{b}^{2}}\right]$ 
$\mathrm{ENL}=\mu_{m}^{2} / \sigma_{m}^{2}$

where $\mu_{m}$ is the mean of the pixel values in ROI, $\sigma_{m}$ is the pixel standard deviation, and $\mu_{b}$ and $\sigma_{b}$ are the pixel mean and standard deviation of a background region of the image, respectively. All measurements are made after logarithmic transformation.

\section{Results and Discussion}

Figure 4 shows the homogeneous phantom experiments using the half- (left column) and full-channel (right column) methods. The first row presents the representative B-scan modulation spectrums in the $\nu$-domain. The half-channel method has a half-space original spectrum which is totally shifted to one side of $\mathrm{dc}$ and has a conjugate term in the negative halfspace as plotted by the solid blue curve in Fig. 4(a); the positive half-space spectrum is equally split into two subbands $\left(N_{H}=2\right)$ using the Gaussian filter bank, as indicated by the dotted red curves. The full-channel method has a full-space original spectrum covering both the negative and positive half-spaces as plotted by the solid blue curve in Fig. 4(b), and each halfspace spectrum is split into subbands (totally $2 N_{H}=4$ in full space) using two filters. Figures 4(c) and 4(d) are the representative structural cross-sections before compounding. The compounded images are presented in Figs. 4(e) and 4(f). The speckle contrasts before compounding are 0.54 and 0.56 in the half- and

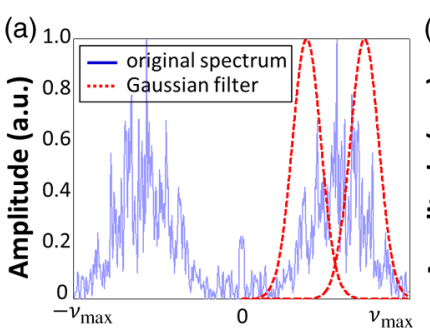

Modulation frequency
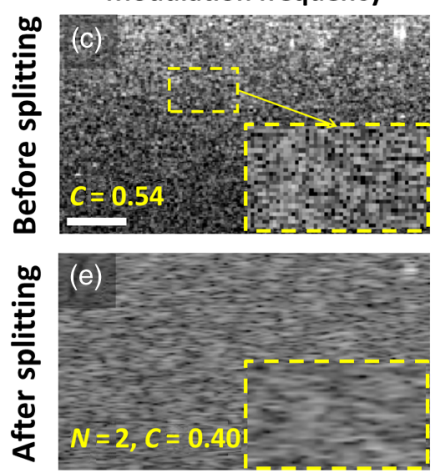

Half-channel

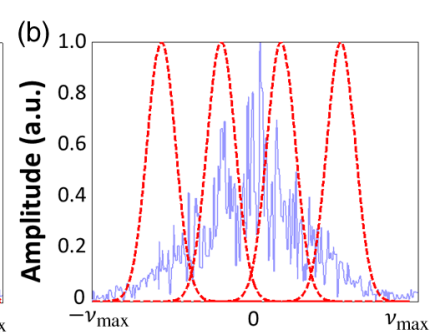

Modulation frequency
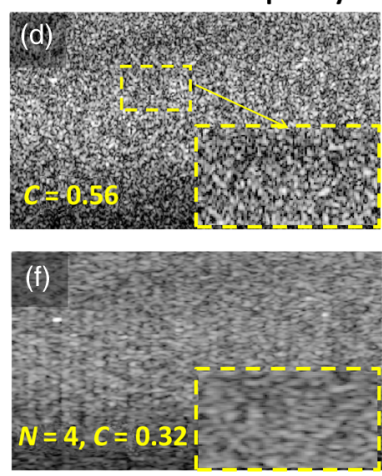

Full-channel
Fig. 4 Homogeneous phantom experiment using the half- (left column) and full-channel (right column) methods. The first row is representative B-scan modulation spectrums in the $\nu$-domain. (a) Halfchannel method has a half-space original spectrum which is totally shifted to one side of dc and has a conjugate term in the negative half-space (solid blue curve), and the dotted red curves indicate two Gaussian filters used. (b) Full-channel method has a full-space original spectrum covering both the negative and positive half-spaces (solid blue curve), and the dotted red curves indicate the Gaussian filter banks with four members used. The second and third rows show the representative structural cross-sections before and after $\left(N_{H}=2\right)$ compounding, respectively. The inserts in (c)-(f) are enlarged views of the rectangular areas. The scale bar: $500 \mu \mathrm{m}$.
Table 1 Comparisons of image-quality metrics for OCT phantom images in half- and full-channel methods.

\begin{tabular}{llccc} 
Methods & & $C$ & $\mathrm{CNR}(\mathrm{dB})$ & $\mathrm{ENL}$ \\
\hline Half-channel & Original & 0.54 & 2.53 & 46.28 \\
& $N_{H}=2$ & 0.40 & 2.82 & 78.13 \\
Full-channel & Original & 0.56 & 2.61 & 48.51 \\
& $2 N_{H}=4$ & 0.32 & 5.03 & 117.82 \\
\hline
\end{tabular}

full-channel methods, respectively, which are in good agreement with the theoretical value of 0.52 . The corresponding speckle contrasts fall to 0.40 with a reduction factor of 1.35 , and 0.32 with a factor of 1.75 after compounding, respectively. The residual correlations between the angle-resolved subimages in the half- and full-channel methods are 0.05 and 0.07 , respectively. It can be perceived from the enlarged views of rectangular areas $(50 \times 80$ pixels $)$ that the angular compounded images exhibit a reduction of speckle noise in both the half- and full-channel methods. Furthermore, compared to the half-channel, the full-channel method offers higher efficiency in speckle reduction.

In the phantom image, the air background region was used to calculate the noise level. The CNR values were averaged over six different ROIs and the ENL values were averaged over three homogeneous regions. The CNR and ENL in both half- and full-channel methods before splitting are in agreement with each other, while these values show greater improvements in the full-channel method with the doubled number of splits $2 N_{H}$ compared to those in half-channel method with the number of splits of $N_{H}$. Table 1 lists the results of the quality metrics $C$, CNR, and ENL.

The changes of speckle contrast $(C)$ versus the number of splits/averages in half-channel $N_{H}$ are plotted in Fig. 5. Generally, a large number of splits/averages leads to a high extent of speckle reduction. The full-channel method exhibits a larger reduction in speckle than the half-channel method because of the doubled number of splits $2 N_{H}$. The measurement has a small deviation from the theoretical curve, which is most likely due to the residual correlation among the angle-resolved subimages.

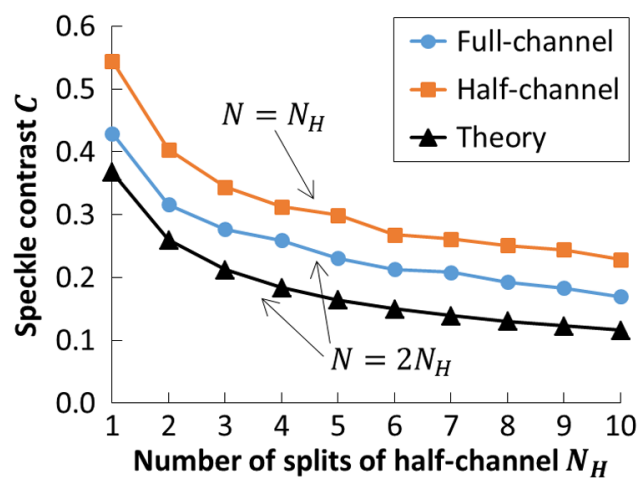

Fig. 5 Changes of speckle contrast $(C)$ versus number of splits/ averages of half-channel $N_{H}$. Squares, half-channel, $N=N_{H}$; circles, full-channel, $N=2 N_{H}$; and triangles, theory, $N=2 N_{H}$. 

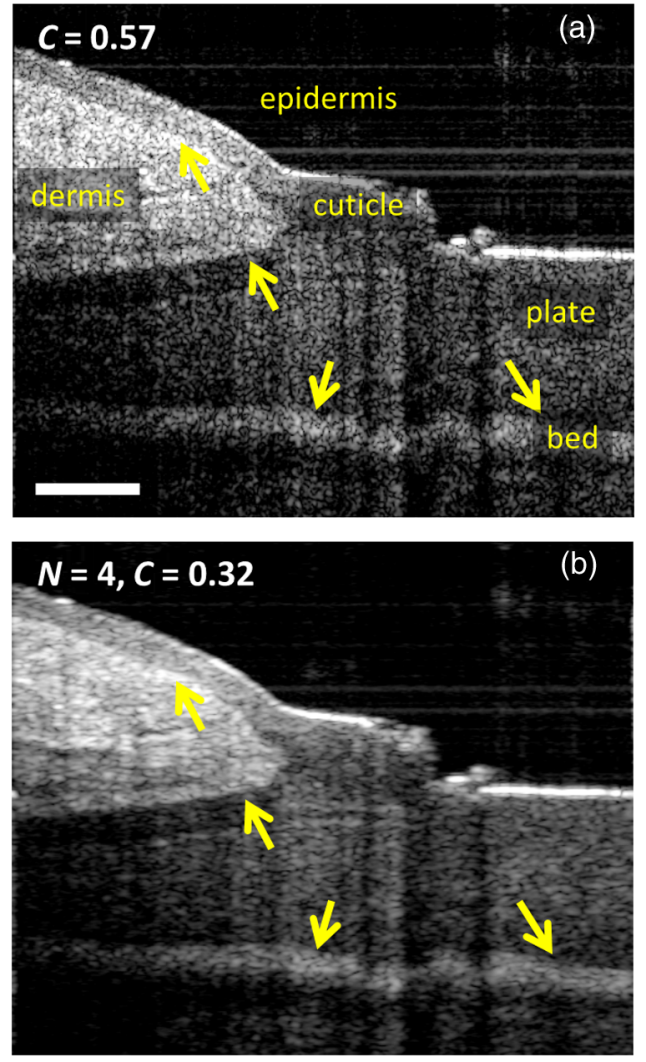

Fig. 6 Representative structural cross-sections of the proximal nail fold of the human thumb in vivo using the full-channel approach (a) before and (b) after compounding $\left(N=2 N_{H}=4\right)$. The yellow arrows indicate the enhanced visibility of layered structures after compounding. The scale bar: $500 \mu \mathrm{m}$.

Figure 6(a) shows the representative structural cross-sections of the proximal nail fold of the thumb from a human volunteer using our proposed full-channel method before compounding. The corresponding image after compounding at $N=2 N_{H}=4$ is presented in Fig. 6(b). The visibility of the boundaries (epidermis-dermis-plate-bed) is obviously enhanced in the compounded images, as indicated by the yellow arrows. According to the speckle contrast calculation in the areas of the nail plate, the full-channel method represents a factor of 1.78 in speckle reduction, which is in agreement with the phantom result 1.75 .

As we know, there exists a tradeoff between the speckle reduction and lateral resolution. ${ }^{4,10}$ As indicated by Eq. (2), a split of the modulation spectrum is equivalent to a division of the beam size, resulting in a decrease of the effective numerical aperture and thereby a loss of lateral resolution. For each channel, taking the assumption that the original probe beam with the diameter of $D$ is equally divided with the same and circle aperture (diameter $d=D / N$ ) along the direction perpendicular to the optical axis, the lateral resolution of each subimage $\delta_{x i}$ can be approximated as ${ }^{19}$

$\delta_{x i}=\frac{8}{k_{0}} \frac{f N}{D}$

where $f$ is the focal length of the objective lens in the sample arm. Here, we define $N / \delta_{x i}$ as the performance factor of speckle reduction $F_{\mathrm{SR}}$
$F_{\mathrm{SR}}=\frac{N}{\delta_{x i}}=\frac{k_{0}}{8} \frac{D}{f}$.

A higher factor $F_{\mathrm{SR}}$ means a larger reduction of speckle noise at a lower cost of lateral resolution. The full-channel method offers more than twice the performance in speckle reduction as the half-channel method because $D_{F}>2 D_{H}$ in practice.

Currently, the modulation spectrum is equally split into $N$ subbands with the same bandwidth and a certain band overlap. However, the modulation spectrum is usually a Gaussian shape, thus the light power is nonuniformly distributed within the subbands. The band overlap is helpful for reducing the loss of signal, but leads to a residual correlation between the subimages which degrades the speckle reduction. Further optimization of the splitting procedure is required by taking full account of these factors.

In the proposed full-channel method, to reconstruct the complex-valued SI, the entirety of sample must be situated on one side of the zero path length, and only half of the depth space (i.e., imaging range) can be used for imaging. Fortunately, the speckle noise appears most frequently in the high-scattering sample, where the penetrating depth is $<2 \mathrm{~mm}$. The SDOCT usually has a half-depth range of $>3 \mathrm{~mm}$ which is sufficient to image high-scattering samples. Therefore, the full-channel method is suitable for most of the applications when speckle reduction is necessary in spite of sacrificing a half-range in the depth space.

\section{Conclusions}

In brief, we have proposed an angular compounding method by full-channel B-scan modulation encoding for OCT speckle reduction. By discarding a half-range in the depth space, the full-channel method allows the negative and positive halfchannels to work in parallel and offers more than twice the performance in speckle reduction compared with the existing half-channel method. The feasibility of the proposed fullchannel approach is validated through both the phantom and in vivo human thumbnail experiments. The proposed method can be readily applied to the existing commercial system without additional system complexity and cost.

\section{Acknowledgments}

This work was supported by the National Natural Science Foundation of China under Grant Nos. 61475143, 11404285, 61335003, 61327007, and 61275196, Zhejiang Provincial Natural Science Foundation of China under Grant No. LY14F050007, Zhejiang Province Science and Technology under Grant No. 2015C33108, the National Hi-Tech Research and Development Program of China under Grant No. 2015AA020515, and the Scientific Research Foundation for Returned Scholars, Ministry of Education of China.

\section{References}

1. D. Huang et al., "Optical coherence tomography," Science 254(5035), 1178-1181 (1991).

2. Y. Ni et al., "Assessment of full-eye response to osmotic stress in mouse model in vivo using optical coherence tomography," J. Ophthalmol. 2015(6), 1-8 (2015).

3. S. Sizmaz et al., "Assessment of tear meniscus with optical coherence tomography in thyroid-associated ophtalmopathy," Curr. Eye Res. 39(4), 323-328 (2014). 
4. J. M. Schmitt, S. H. Xiang, and K. M. Yung, "Speckle in optical coherence tomography," J. Biomed. Opt. 4(1), 95-105 (1999).

5. M. Bashkansky and J. Reintjes, "Statistics and reduction of speckle in optical coherence tomography," Opt. Lett. 25(8), 545-547 (2000).

6. D. Alonso-Caneiro, S. A. Read, and M. J. Collins, "Speckle reduction in optical coherence tomography imaging by affine-motion image registration," J. Biomed. Opt. 16(11), 116027 (2011).

7. Y. Du et al., "Speckle reduction in optical coherence tomography images based on wave atoms," J. Biomed. Opt. 19(5), 056009 (2014).

8. M. Pircher et al., "Speckle reduction in optical coherence tomography by frequency compounding," J. Biomed. Opt. 8(3), 565-569 (2003).

9. N. Iftimia, B. E. Bouma, and G. J. Tearney, "Speckle reduction in optical coherence tomography by "path length encoded" angular compounding," J. Biomed. Opt. 8(2), 260-263 (2003).

10. H. Wang and A. M. Rollins, "Speckle reduction in optical coherence tomography using angular compounding by B-scan Doppler-shift encoding," J. Biomed. Opt. 14(3), 030512 (2009).

11. B. J. Huang et al., "Speckle reduction in parallel optical coherence tomography by spatial compounding," Opt. Laser Technol. 45, 69-73 (2013).

12. E. Gotzinger et al., "Speckle noise reduction in high speed polarization sensitive spectral domain optical coherence tomography," Opt. Express 19(15), 14568-14585 (2011).
13. B. F. Kennedy et al., "Speckle reduction in optical coherence tomography by strain compounding," Opt. Lett. 35(14), 2445-2447 (2010).

14. L. An and R. K. Wang, "Use of a scanner to modulate spatial interferograms for in vivo full-range Fourier-domain optical coherence tomography," Opt. Lett. 32(23), 3423-3425 (2007).

15. P. Li et al., "Single-shot angular compounded optical coherence tomography angiography by splitting full-space B-scan modulation spectrum for flow contrast enhancement," Opt. Lett. 41(5), 1058-1061 (2016).

16. S. S. Gao et al., "Optimization of the split-spectrum amplitudedecorrelation angiography algorithm on a spectral optical coherence tomography system," Opt. Lett. 40(10), 2305-2308 (2015).

17. Y. Jia et al., "Split-spectrum amplitude-decorrelation angiography with optical coherence tomography," Opt. Express 20(4), 4710-4725 (2012).

18. D. C. Adler, T. H. Ko, and J. G. Fujimoto, "Speckle reduction in optical coherence tomography images by use of a spatially adaptive wavelet filter," Opt. Lett. 29(24), 2878-2880 (2004).

19. W. Drexler and J. G. Fujimoto, Optical Coherence Tomography: Technology and Applications, Springer, Berlin, Heidelberg (2008).

Biographies for the authors are not available. 